\title{
Association Between Posttraumatic Stress Disorder Following Myocardial Infarction and Liver Enzyme Levels: A Prospective Study
}

\author{
Roland von Känel • Chiara C. Abbas · \\ Stefan Begré • Marie-Louise Gander • \\ Hugo Saner · Jean-Paul Schmid
}

Received: 2 June 2009/Accepted: 1 December 2009/Published online: 24 December 2009

(C) Springer Science+Business Media, LLC 2009

\begin{abstract}
Background Research in rodents demonstrated that psychological stress increases circulating levels of alanine transaminase, aspartate transaminase, and alkaline phosphatase reflecting liver injury. Moreover, chronic posttraumatic stress disorder and transaminases predicted coronary heart disease.

Aims To investigate the hypothesis that severity of posttraumatic stress disorder following myocardial infarction would prospectively relate to liver enzymes.

Methods Study participants were 24 patients (mean $59 \pm 7$ years, $79 \%$ men) with an interviewer-rated diagnosis of posttraumatic stress disorder caused by an index myocardial infarction $3 \pm 3$ months before. After a mean follow-up of $26 \pm 6$ months, patients had a clinical interview to reassess posttraumatic stress disorder severity, a medical history, and blood collected to determine liver enzymes.

Results Total posttraumatic stress disorder symptoms assessed at study entry prospectively predicted plasma levels of alanine transaminase $(r=.47, p=.031)$ and alkaline phosphatase $(r=.57, p=.004)$, but not of aspartate transaminase $(p=.15)$, controlling for follow-up duration and antidepressant use. Total posttraumatic stress disorder symptoms assessed at follow-up were associated
\end{abstract}

R. von Känel $(\bowtie)$ · C. C. Abbas · S. Begré · M.-L. Gander Department of General Internal Medicine, Division of Psychosomatic Medicine, Inselspital, Bern University Hospital, and University of Bern, 3010 Bern, Switzerland

e-mail: roland.vonkaenel@insel.ch

R. von Känel · C. C. Abbas · H. Saner · J.-P. Schmid Swiss Cardiovascular Center Bern, Cardiovascular Prevention and Rehabilitation, Inselspital, Bern University Hospital, and University of Bern, Bern, Switzerland with alanine transaminase $(r=.72, p=.004)$, aspartate transaminase $(r=.60, p=.018)$, and alkaline phosphatase $(r=.64, p=.001)$ in the 16 patients who had maintained diagnostic posttraumatic stress disorder, but not in all 24 patients.

Conclusions The severity of posttraumatic stress disorder following myocardial infarction was associated with mild increase in liver enzyme levels, suggesting that chronic psychological stress relates to hepatic damage in humans. This might help to explain the previously observed increased cardiovascular risk in chronically traumatized individuals.

Keywords Cardiovascular diseases - Liver .

Myocardial infarction - Posttraumatic stress disorder .

Psychological stress $\cdot$ Transaminases

\section{Introduction}

While alanine transaminase (ALT), aspartate transaminase (AST), and alkaline phosphatase (ALP) are enzymes located in tissues throughout the body, they are most often increased as a result of liver injury, even in asymptomatic patients [1]. As markers of liver damage, elevated levels of ALT and AST typically reflect hepatocellular disease and increased ALP levels are typically indicative for hepatobiliary disease $[2,3]$.

Experimental exposure to psychological stress impacts liver structure in rodents, perhaps most evident in animals with a coexistent liver or cardiovascular disease (CVD). For instance, an aggressive encounter in male mice caused increased plasma activities of ALT and AST after $3 \mathrm{~h}$ as well as necrotic lesions of the liver parenchyma and damage to hepatocytes $8 \mathrm{~h}$ later [4]. Electron microscopy 
showed autophagy of the liver compatible with hepatic damage 2 days after exposure of rats to a variety of acute stressors, including restraint stress [5]. Repeated coldimmobilization stress for 5 days increased serum ALP levels and was accompanied by liver failure in cardiomyopathic hamsters but not in healthy control hamsters [6]. Oxidative damage to nuclear DNA of hepatocytes was found in rats after 4 days of witnessing their mates being electronically shocked [7]. To conclude, a recent review proposed that psychological stress promotes a hepatic inflammatory response that might contribute to the risk of developing and aggravating several liver diseases [8].

Very few studies have actually addressed the potential role of psychological stress in liver diseases in humans. In patients with chronic alcoholic hepatitis, life stress was associated with fibrosis and inflammatory changes [9]. In Japanese workers, chronic job stress was associated with elevated ALT levels, even when adjustment was made for demographic, metabolic, and life style factors, including alcohol intake [10]. Whether chronic psychological stress prospectively predicts liver injury and elevation in liver enzymes, respectively, has not been investigated in humans.

Posttraumatic stress disorder (PTSD) is a debilitating form of chronic psychological stress that develops after exposure to a traumatic event to which a person responded with fear, helplessness, or horror [11]. Traditionally, PTSD has been related to natural disasters and man-made trauma such as combat exposure and sexual assaults [11]. However, it is increasingly acknowledged that PTSD may occur in relation to medical diagnoses and procedures such as in the context of multiple physical trauma, severe burns, cancer, and heart diseases [12-15]. Specifically, after an acute myocardial infarction (MI), about 10\% of patients develop either full or subthreshold PTSD meeting Diagnostic and Statistical Manual of Mental Disorders (DSM-IV) criteria [16]. In addition to having experienced the life-threatening heart attack as traumatic, the patients must have three distinct types of symptoms consisting of re-experiencing the MI, avoidance of reminders of the MI, and hyperarousal such as irritability and sleep disturbances for at least 1 month [11, 17]. Posttraumatic stress symptoms profoundly impact daily functioning and may persist for many years [11]. Of the 24 post-MI patients with DSM-IV PTSD also investigated in the present study, two-thirds still endorsed diagnostic PTSD, on average, 2 years later [18].

Posttraumatic stress disorder is associated with poor physical health and particularly CVD [17, 19]. Military veterans with PTSD initially free of CVD run a greater risk for early age heart disease mortality [20]. The risk for cardiovascular re-admissions was increased in patients who had developed PTSD following MI, too [21]. A positive screen for military sexual trauma was previously associated with an increased risk of liver disease in US veterans [22].
However, whether PTSD relates to liver diseases has to our knowledge not been investigated.

Carotid atherosclerosis is an inflammatory disease that has been associated with non-alcoholic fatty liver disease (NAFLD) [23] for which elevated levels of liver transaminases and ALP are markers [24]. Biomarkers of systemic inflammation predict coronary heart disease (CHD) [25], showed direct associations with ALT and ALP [26, 27], and were also elevated in relation to symptoms of PTSD $[17,28]$. Elevated ALT predicted incident CHD after 10 years [29] and also coronary atherosclerosis in patients with suspicious CHD, even after controlling for inflammation and components of the metabolic syndrome [30].

Taken together, the above research implies that chronic psychological stress might contribute to liver injury and transaminases increase by kindling a hepatic and systemic inflammatory response setting the stage for the initiation and progression of atherosclerosis. Such psychopathophysiologic mechanisms might explain some of the excess risk for incident CHD and recurrent CVD events in chronically traumatized victims and post-MI patients, respectively. We aimed to verify part of this notion to ultimately better understand the biology of PTSD and its physical health consequences. We studied the hypothesis that diagnostic PTSD, which had developed after MI, would prospectively predict plasma levels of ALT, AST, and ALP after a mean follow-up of 2 years.

\section{Materials and Methods}

\section{Study Participants}

The study protocol was part of the ongoing longitudinal Swiss Heart and Mind Study and approved by the ethics committee of the State of Bern, Switzerland. All participants provided written informed consent. The recruitment procedure has previously been detailed with regard to the identification of the 24 patients with PTSD following an index MI investigated in the present study $[16,18]$. In brief, all patients had been referred to the Department of Cardiology, University of Bern, Switzerland, with a verified ST elevation or non-ST elevation MI according to previous guidelines [19] and had left ventricular ejection fraction (LVEF) determined during coronary angiography. On average, $3 \pm 3$ months (range 1-14) after the index MI, the 24 patients were mailed home the German version of the self-rated posttraumatic diagnostic scale (PDS) [31] asking about the 17 PTSD symptoms according to DSM-IV criteria [32]. Based on meeting a predefined cut-off of PTSD symptom severity on the PDS, they were invited to undergo a structured interview applying the DSM-IV Clinicianadministered PTSD scale (CAPS) taking place on average 
$4.2 \pm 3.2$ months (range 2-16) after the index MI. According to the CAPS interview, eight patients had full PTSD and 16 patients had subthreshold PTSD (cf. below). Individual PTSD symptom clusters from the entry interview, but not the total PTSD symptom score, were missing in one participant.

\section{Follow-Up Investigation}

For the follow-up investigation presented here, the 24 patients were re-invited to the psychocardiology research laboratory of the Cardiac Prevention and Rehabilitation, University of Bern, Switzerland, between 1 and 3 years after the initial diagnosis of PTSD. The investigation took $11 / 2 \mathrm{~h}$ and was scheduled to start after lunch between 12:30 p.m. and 2:00 p.m. Patients were asked to follow their daily routine on the test day and to consume no heavy meal for lunch. They were also asked to abstain from smoking, alcoholic beverages, and sports activities starting on the morning of the test day.

Upon arrival at the laboratory, participants first underwent a structured medical history about past and current diseases, current prescribed medications, and health habits. Specifically, patients were asked about the number of alcoholic drinks consumed in an average week, their sports activities in hours performed in an average week, and whether they currently smoked. After a resting period of at least 15 min during which patients were sitting, blood pressure (BP) was measured using sphygmomanometry. We recorded BP three times with an interval of 5 min between readings and computed the average of the three readings to obtain screening systolic and diastolic BP. We measured height and weight to compute the body mass index (BMI), collected blood samples for the laboratory analyses and performed the CAPS interview in the next sequence.

\section{Clinician-Administered PTSD Scale}

The clinician-administered PTSD Scale (CAPS) is a structured psychometric interview allowing for a diagnosis of PTSD as defined in the DSM-IV [33]. We applied the German version of the CAPS showing good internal consistency for the severity score of all 17 items of PTSD (Cronbach's $\alpha=.88-.92$ ) and for each of the three PTSD symptom clusters $(\alpha=.73-.88)$ [34]. The frequency and intensity of each of the 17 PTSD symptoms are quantified on a five-point scale ranging between e.g., "never" (0 points) to "almost always" (4 points). A particular symptom is given when rated with a frequency of $\geq 1$ point and an intensity of $\geq 2$ points. To meet diagnostic criteria for a particular symptom cluster, criterion B (re-experiencing subscale) requires one of five symptoms, criterion $\mathrm{C}$ (avoidance subscale) requires three of seven symptoms, and criterion D (hyperarousal subscale) requires two of five symptoms. The CAPS symptom subscales provide a continuous measure of PTSD symptom severity. Overall severity of PTSD is obtained by adding symptom scores of criteria B plus C plus D to a total PTSD symptom score. Full (i.e., syndromal) PTSD meets criteria B, C, and D, and subthreshold (i.e., subsyndromal PTSD) meets criterion B plus either C or D [35].

\section{Laboratory Analyses}

Venous blood samples were collected in appropriate tubes and analyzed immediately within the clinical routine at the Institute of Clinical Chemistry, University Hospital Bern, Switzerland, applying standard laboratory procedures (certified ISO/ICE). A Modular P800 system with ion selective electrodes and Cobas standard test kits (all Roche Diagnostics, Rotkreuz, Switzerland) were used to determine levels of liver enzymes and lipids in plasma. Glycosylated hemoglobin A1c (HbA1c) was measured by high-performance liquid chromatography on a HLC-723 G7 analyzer (Tosoh Corporation, Tokyo, Japan).

\section{Statistical Analysis}

Data analysis used SPSS 15.0 statistical software package (SPSS Inc. Chicago, IL) with significance level set at $p<.05$ (two-tailed). Because several variables were not normally distributed, we applied non-parametric statistics throughout. For clarity, all data are provided in original units, except in figures which show transformed values. Specifically, we used the Mann-Whitney $U$ test and Fisher's exact test to test for differences between two groups in continuous and categorical data, respectively. The Spearman rank test was used to estimate the crude correlation between two variables. Blom normal score transformed variables were used to compute partial correlation coefficients with $95 \%$ confidence intervals. Given the sample size, we allowed for a maximum of two control variables a priori in order to prevent overfitting of statistical models [36]. These were the duration of follow-up and use of antidepressant medication if being significantly associated with liver enzymes in the crude correlation analysis. A longer duration of PTSD might be expected to have a greater impact on liver function and patients with more severe PTSD might more readily be prescribed antidepressants, which, in turn, may cause elevation of liver enzymes. We did not correct $p$-values for multiple tests because in our case of a preestablished hypothesis (i.e., direct relationship between PTSD severity and enzyme levels), this might deem truly important associations insignificant, thereby misleading future studies in a still nascent area of research [37]. 


\section{Results}

\section{Subject Characteristics}

Table 1 shows the demographic, metabolic, and health behavior variables of the 24 patients at follow-up. Plasma levels of liver enzymes were, on average, in the normal laboratory range. However, taking into account age and sex of individual patients, three exceeded the upper normal reference range for both ALT and AST, whereas one exceeded the upper normal reference range for AST only. Table 2 shows the medication list of study participants at follow-up. Particularly, all patients had lipid lowering therapy (statins in 23 of cases) and four patients were prescribed antidepressants.

During the follow-up period, no acute cardiovascular events had occurred requiring hospital admission. Three patients had undergone elective coronary stenting. Two patients who were also on antidepressants had psychotherapy without trauma-specific elements.

\section{Associations with Liver Enzymes}

Transaminases correlated with each other $(r=.62$, $p=.002$ ), but not significantly with ALP. Alanine transaminase correlated with age $(r=-.50, p=.015)$ and was higher in patients with a history of diabetes than in those without $(53.0 \pm 29.7$ vs. $26.9 \pm 12.2 \mathrm{U} / 1, p=.026)$. No other associations emerged between any other demographic, metabolic, and life style factor shown in Table 1 on the one hand and ALT, AST, and ALP levels on the other

Table 1 Characteristics of the 24 myocardial infarction patients

\begin{tabular}{ll}
\hline Male $(\%)$ & 79 \\
Age (years) & $58.6 \pm 6.7(45-74)$ \\
Left ventricular ejection fraction $(\%)$ & $52.1 \pm 9.4(25-65)$ \\
Body mass index $\left(\mathrm{kg} / \mathrm{m}^{2}\right)$ & $27.7 \pm 3.7(20.1-36.8)$ \\
Total cholesterol $(\mathrm{mmol} / \mathrm{l})$ & $4.0 \pm .9(2.2-6.5)$ \\
Triglycerides (mmol/l) & $1.7 \pm 1.1(.5-5.9)$ \\
Hemoglobin A1c $(\%)$ & $6.0 \pm .8(5.3-9.0)$ \\
Type II diabetes $(\%)$ & 13 \\
Systolic blood pressure (mmHg) & $124 \pm 15(99-150)$ \\
Diastolic blood pressure (mmHg) & $83 \pm 9(55-100)$ \\
Treated for systemic hypertension $(\%)$ & 96 \\
Alcohol consumption (drinks/week) & $5.0 \pm 6.2(0-21)$ \\
Sports activities (h/week) & $4.2 \pm 3.0(0-9)$ \\
Current smoker $(\%)$ & 21 \\
Alanine transaminase (U/l) & $30.3 \pm 17.0(11-87)$ \\
Aspartate transaminase (U/l) & $30.2 \pm 9.1(17-56)$ \\
Alkaline phosphatase (U/l) & $64.2 \pm 20.7(24-108)$ \\
\hline
\end{tabular}

Values are given as means \pm SD with range or percentages
Table 2 Medication list of the 24 study participants

\begin{tabular}{lr}
\hline Statins (\%) & 96 \\
Other lipid lowering drugs (\%) & 12 \\
Aspirin (\%) & 96 \\
Clopidogrel (\%) & 29 \\
Phenprocoumon (\%) & 4 \\
Insulin (\%) & 4 \\
Oral antidiabetics (\%) & 4 \\
Beta blockers (\%) & 87 \\
Angiotensin converting enzyme inhibitors (\%) & 42 \\
Angiotensin II receptor blockers (\%) & 25 \\
Calcium antagonists (\%) & 21 \\
Diuretics (\%) & 25 \\
Amiodarone (\%) & 8 \\
Levothyroxine (\%) & 8 \\
Tamoxifen (\%) & 4 \\
Pain medication (\%) & 25 \\
Antidepressants (\%) & 17 \\
Benzodiazepines (\%) & 4 \\
\hline
\end{tabular}

Values are given as percentages referring to the population of all 24 patients

(all $p$-values $>.05$ ). In particular, no association emerged between LVEF and liver enzymes ( $p$-values $>.59$ ). In terms of medication, the four patients on antidepressants had higher plasma levels of ALT $(51 \pm 27$ vs. $26 \pm 12 \mathrm{U} / \mathrm{l}$, $p=.009)$ and of AST (42 \pm 12 vs. $28 \pm 6 \mathrm{U} / \mathrm{l}, p=.029)$ than the 20 patients not taking antidepressants. Liver enzymes showed no significant correlation with any other medication category listed in Table 2 (all $p$-values $>.05$ ).

\section{Posttraumatic Stress Symptoms}

The mean follow-up was $26.2 \pm 6.5$ months (range 12-36), during which there was a significant decrease in the total PTSD symptom score $(53.0 \pm 21.6$ vs. $36.3 \pm 26.5$, $p=.001)$, re-experiencing symptoms $(18.3 \pm 7.5$ vs. $10.6 \pm 8.5, p<.001)$, avoidance symptoms $(18.7 \pm 9.8$ vs. $13.8 \pm 13.1, p=.015)$, but not in hyperarousal symptoms $(16.7 \pm 7.6$ vs. $13.2 \pm 8.0, p<.06)$. Of the 24 postMI patients with a diagnosis of PTSD at study entry, 16 still fulfilled the diagnostic criteria for PTSD at follow-up.

Posttraumatic Stress Symptoms and Liver Enzymes

\section{PTSD Symptoms at Study Entry and Liver Enzymes}

With adjustment to the duration of follow-up and antidepressant use, the total score of PTSD symptoms assessed at study entry significantly predicted ALT (Fig. 1a) and ALP (Fig. 3a) to explain 22 and $32 \%$ of the respective variance 

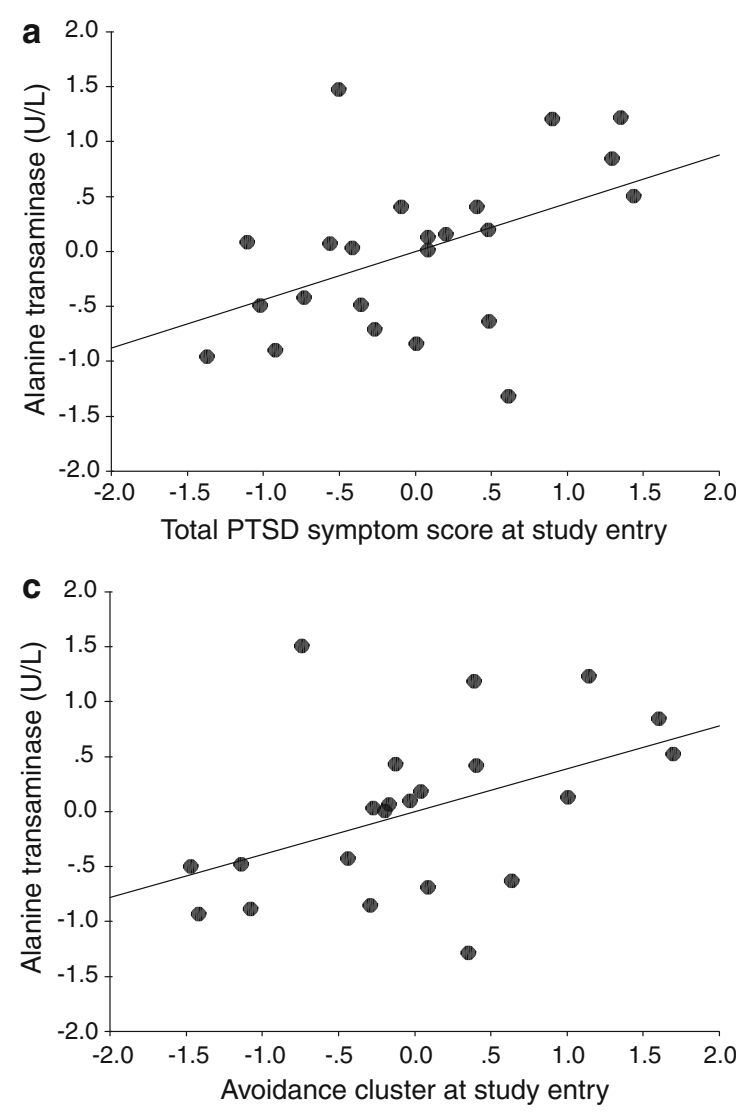

Fig. 1 Scatter plot with fit line showing the significant associations between alanine transaminase $(A L T)$ and study entry scores for total PTSD symptoms (a; $p=.031)$, re-experiencing symptoms

(Table 3). In contrast, the total PTSD symptom score at entry was not a significant predictor of AST $(p=.15)$.

Table 3 further shows that of the individual PTSD symptom clusters assessed at study entry, re-experiencing symptoms significantly predicted ALT (Fig. 1b) and ALP (Fig. 3b), explaining 27 and $26 \%$ of the respective variance. Avoidance symptoms significantly predicted ALT (Fig. 1c), AST (Fig. 2a), and ALP (Fig. 3c) to account for 21, 19, and $40 \%$ of respective variances. Eventually, hyperarousal symptoms significantly explained $19 \%$ of the variance of both AST (Fig. 2b) and ALP (Fig. 3d).

\section{PTSD Symptoms at Follow-Up and Liver Enzymes}

Neither the total PTSD symptom score nor individual symptom clusters assessed at follow-up were significantly associated with any liver enzyme in all 24 patients with adjustment for duration of follow-up and antidepressant use (Table 3). However, in the subgroup of the 16 patients who still had diagnostic PTSD in the follow-up interview, the total follow-up score of PTSD symptoms correlated with $\operatorname{ALT}(p=.004)$, AST $(p=.018)$, and $\operatorname{ALP}(p=.001)$, taking into account the duration of follow-up. Symptom

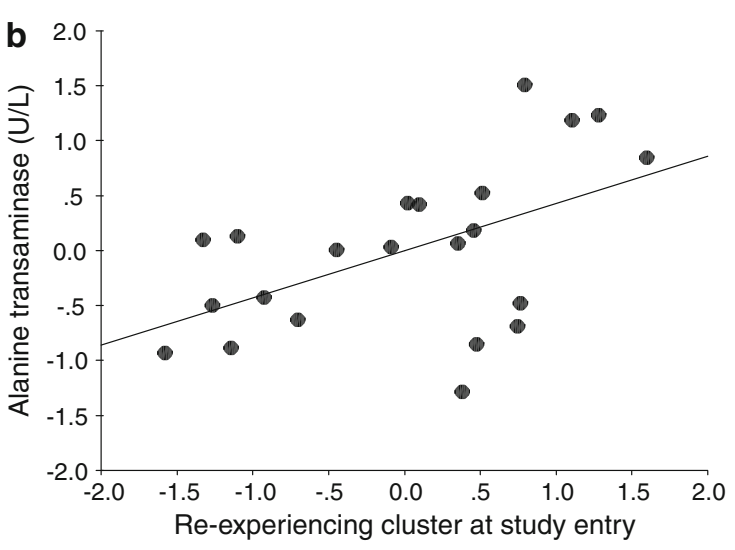

(b; $p=.018)$, and avoidance symptoms (c; $p=.043)$. Adjustment was made for the duration of follow-up and antidepressant use (yes vs. no). Values are given as normal scores

clusters of avoidance ( $p$-values $<.032$ ) and of hyperarousal ( $p$-values <.027) were both associated with each liver enzyme; the re-experiencing cluster was also associated with ALT $(p=.018)$, but not with AST $(p=.27)$ and ALP $(p=.53)$. The analysis in the 16 patients with diagnostic PTSD did not adjust for the use of antidepressants because only two patients were on these medications.

\section{Discussion}

We showed that the severity of PTSD, which had developed in the aftermath of an acute MI, was a significant predictor of plasma levels of ALT and ALP assessed, on average, 2 years after the diagnosis of PTSD. The analysis adjusted for the duration of follow-up and thereby accounted for the possibility that the mere duration of PTSD rather than its severity would impact liver function and structure. We also adjusted for antidepressants which, as seen in our patient group, are well known to increase transaminases levels. In contrast to ALT and ALP, we did not find that the severity of PTSD was a prospective predictor of AST. 
Table 3 Associations between posttraumatic stress symptom scores and liver enzymes

\begin{tabular}{|c|c|c|c|}
\hline & Alanine transaminase & Aspartate transaminase & Alkaline phosphatase \\
\hline \multicolumn{4}{|c|}{ Entry scores (all patients; $n=24$ ) } \\
\hline PTSD total score & $.47(.08-.74)^{*}$ & $.32(-.10-.65)$ & $.57(.22-.80)^{* *}$ \\
\hline Re-experiencing cluster & $.52(.15-.77)^{*}$ & $.16(-.27-.53)$ & $.51(.14-.77)^{*}$ \\
\hline Avoidance cluster & $.46(.07-.74)^{*}$ & $.44(.04-.72)^{*}$ & $.63(.31-.83)^{* *}$ \\
\hline Hyperarousal cluster & $.43(.03-.72)$ & $.44(.04-.72)^{*}$ & $.44(.04-.72)^{*}$ \\
\hline \multicolumn{4}{|c|}{ Follow-up scores (all patients; $n=24$ ) } \\
\hline PTSD total score & $.07(-.35-.46)$ & $.002(-.41-.41)$ & $.23(-.19-.58)$ \\
\hline Re-experiencing cluster & $.05(-.37-.45)$ & $-.08(-.47-.34)$ & $.07(-.35-.46)$ \\
\hline Avoidance cluster & $-.03(-.43-.38)$ & $.10(-.32-.49)$ & $.28(-.14-.62)$ \\
\hline Hyperarousal cluster & $.24(-.18-.59)$ & $.04(-.38-.44)$ & $.33(-.08-.65)$ \\
\hline \multicolumn{4}{|c|}{ Follow-up scores (patients with PTSD at follow-up; $n=16$ ) } \\
\hline PTSD total score & $.72(.35-.90)^{* *}$ & $.60(.15-.85)^{*}$ & $.64(.21-.86)^{*}$ \\
\hline Re-experiencing cluster & $.61(.17-.85)^{*}$ & $.30(-.23-.70)$ & $.18(-.35-.63)$ \\
\hline Avoidance cluster & $.58(.12-.84)^{*}$ & $.65(.23-.87)^{* *}$ & $.69(.29-.89)^{* *}$ \\
\hline Hyperarousal cluster & $.79(.49-.93)^{* *}$ & $.57(.10-.84)^{*}$ & $.60(.15-.85)^{*}$ \\
\hline
\end{tabular}

The columns show the partial correlation coefficients (95\% confidence interval) with significance level after adjustment for the duration of follow-up. Alanine transaminase and aspartate transaminase were additionally adjusted for antidepressant use in all 24 patients with PTSD at study entry

PTSD posttraumatic stress disorder

Significance level: $* p<.05, * * p<.01$

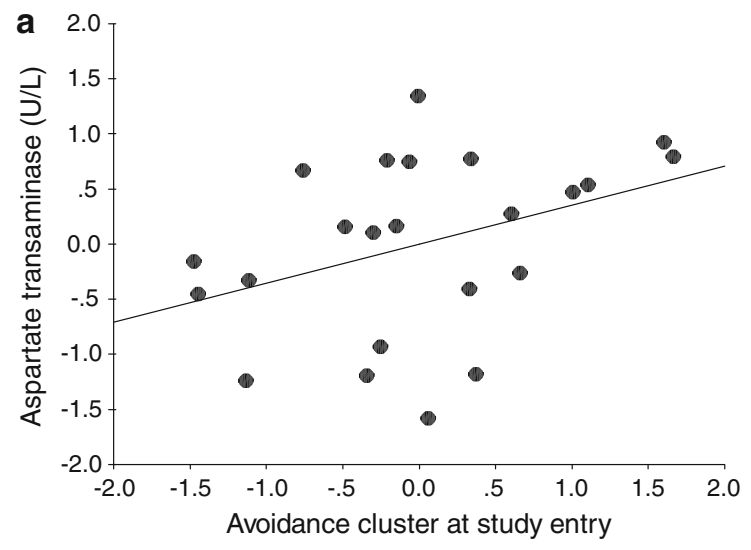

Fig. 2 Scatter plot with fit line showing the significant associations between aspartate transaminase (AST) and study entry scores for symptoms of avoidances $(\mathbf{a} ; p=.049)$ and of hyperarousal

The analysis of individual symptom clusters revealed that the non-significant relationship between PTSD severity at entry and AST was for the most part accounted for by the lack of an association between re-experiencing symptoms and AST. Re-experiencing symptoms predicted ALT and ALP, hyperarousal symptoms were predictive for AST and ALP, and symptoms of avoidance predicted plasma levels of all enzymes. The correlation coefficients of the relationships between the various PTSD symptom scores and enzyme levels were quite similar in absolute terms. Therefore, we feel it premature to propose any symptom

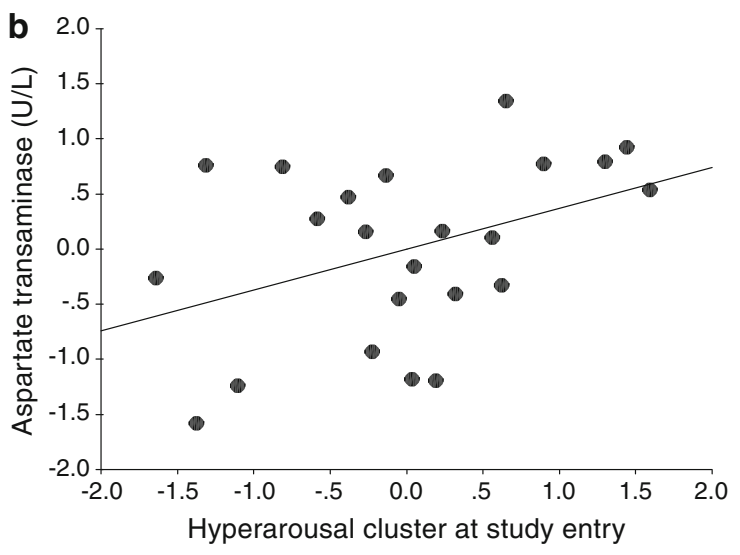

(b; $p=.044)$. Adjustment was made for the duration of follow-up and antidepressant use (yes vs. no). Values are given as normal scores

cluster to be a more reliable predictor of liver enzyme levels than any other. Given the relatively small sample, a type II error of failing to observe a true relationship also cannot be ruled out. Accordingly, there were considerably wide confidence intervals around the observed partial correlation coefficients. Nevertheless, variances in plasma levels of liver enzymes explained by the various PTSD scores were between 19 and $40 \%$, suggesting that these relationships were not only statistically but also clinically meaningful.

As previously reported, while all PTSD symptom scores except hyperarousal had significantly decreased over time, 

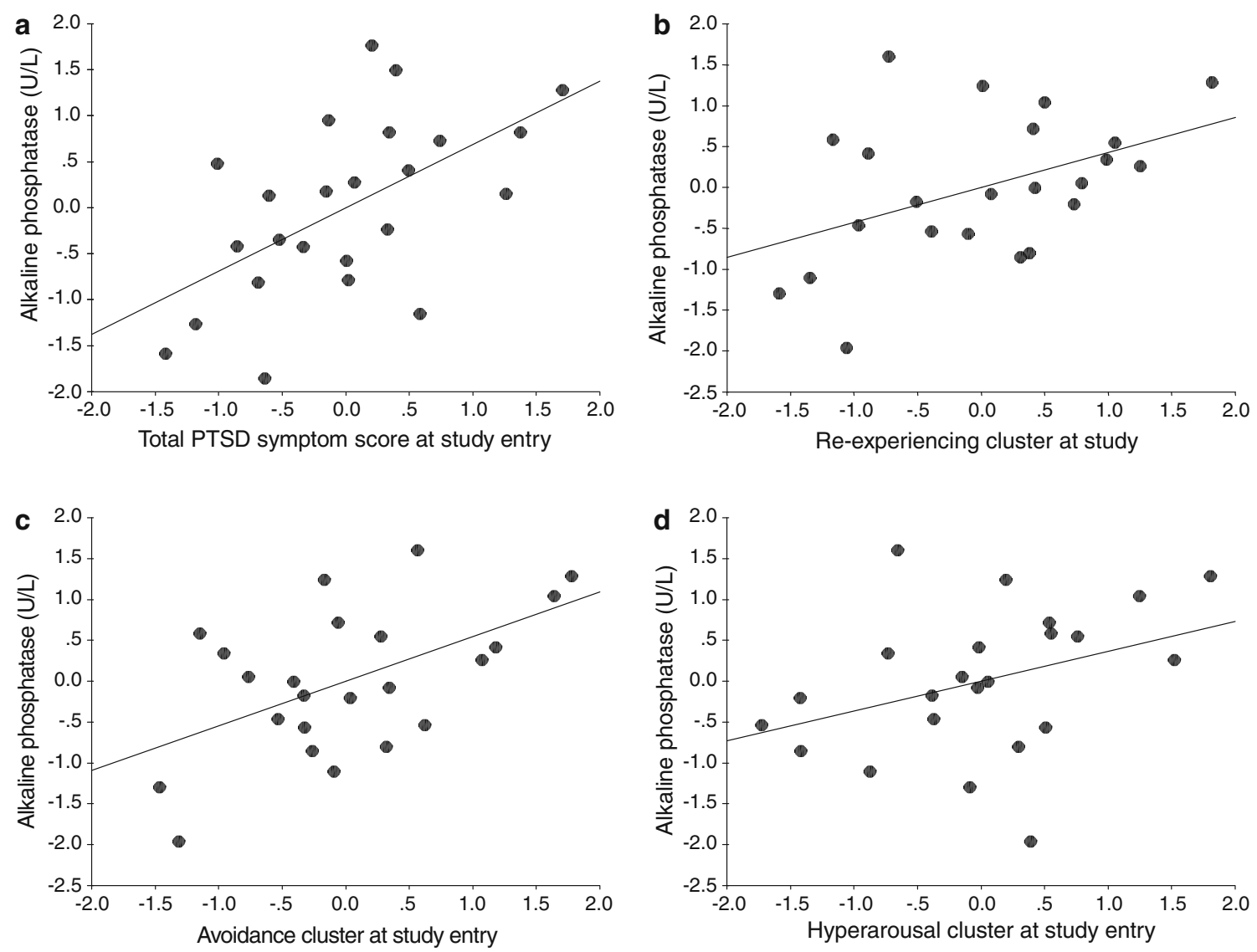

Fig. 3 Scatter plot with fit line showing the significant association between alkaline phosphatase $(A L P)$ and study entry scores for total PTSD symptoms $(\mathbf{a} ; \quad p=.004)$, re-experiencing symptoms

$(\mathbf{b} ; p=.016)$, avoidance symptoms $(\mathbf{c} ; p=.002)$, and hyperarousal symptoms $(\mathbf{d} ; p=.040)$. Adjustment was made for the duration of follow-up. Values are given as normal scores

two-thirds of post-MI patients still had diagnostic PTSD at follow-up, which indicates that PTSD related to MI is a considerably persistent stress disorder [18]. Pertinent to the present study, follow-up scores of total PTSD symptoms and of virtually all individual PTSD symptom clusters showed only a significant association with ALT, AST, and ALP in the two-thirds of patients who maintained PTSD. This suggests that even though PTSD severity waned over time, with some individuals even remitting, the long-term effect of an initial diagnosis of PTSD on liver enzymes was sustained. In contrast, PTSD symptomatology had to fulfill criteria of diagnostic PTSD at follow-up in order to reveal a steady-state association between PTSD severity and liver enzymes. However, PTSD patients have a greater degree of sympathetic nervous system activation [11]. In rats, transaminases were significantly increased $3 \mathrm{~h}$ after acute psychological stress [4]. We can therefore not rule out the possibility that anticipatory appraisal of the CAPS interview as overly threatening might have elicited an increase in liver enzymes in relation to PTSD severity.

Our finding of PTSD predicting circulating levels of hepatic enzymes are in accordance with considerably

consistent literature about psychological stress causing liver injury and an increase in hepatic enzymes in rodents [4-7]. Our data also concur with cross-sectional associations between chronic life stress and the degree of chronic hepatitis and ALT levels in humans [9, 10]. Although not investigated in our study, PTSD might provoke hepatic inflammation and liver injury primordially through peripheral effectors of the stress response, namely catecholamines and glucocorticoids. Noradrenergic dysregulation plays a major role in the initiation and maintenance of hyperarousal and reexperiencing symptoms of PTSD [38]. Moreover, military veterans with PTSD showed increased circulating levels of norepinephrine [39] and greater increase in plasma norepinephrine to trauma-specific stimuli [40]. In rodents, footshock stress enhanced chemically induced liver damage; this effect was partially mediated via released catecholamines [41]. Immobilization stress increased interleukin (IL)-6 production in hepatocytes and also elevated plasma IL-6 levels [42]. Hepatocytes and hepatic nonparenchymal cells incubated with norepinephrine in vitro produced IL- 6 and IL- $1 \beta$, respectively [43]. In addition to catecholaminergic hyperactivity, 
PTSD patients show a hypocortisolism consequent to a dysregulated hypothalamic-pituitary-adrenal (HPA) axis $[11,44]$. Glucocorticoids inhibit the production of proinflammatory cytokines by liver macrophages, supporting the assumption that endogenous glucocorticoid deficiency accompanying psychological stress might promote macrophage production of IL-1, IL-6, and tumor necrosis factor- $\alpha$ [45]. Taken together, increased sympathetic outflow to the liver and hypocortisolism provide plausible mechanisms potentially underlying higher levels of liver enzymes in our post-MI patients with more severe PTSD.

It has been proposed that mild increases in liver enzyme levels indicate systemic inflammation that emanates from hepatic inflammation and particularly NAFLD [26]. Systemic inflammation such as related to NAFLD is key to the extent and severity of atherosclerosis [46]. Even when controlling for metabolic factors, NAFLD was an independent predictor of CVD, suggesting its unique role as a cardiometabolic risk factor [47]. The probability of histologically verified NAFLD increases with higher levels of ALT [48], which, in turn, was predictive for CHD [29, 30]. Except for a history of diabetes, metabolic factors and alcohol consumption did not significantly correlate with liver enzymes in our patients. Since diabetes was present in only three subjects, it was inappropriate to test whether diabetes would mediate the relationship between PTSD and ALT levels. On the whole, we found that the increase in liver enzymes related to PTSD was for the most part unrelated to metabolic factors, suggesting that elevated ALT could be a biomarker of increased cardiovascular risk related to PTSD in post-MI patients [21].

Our study has several limitations. The sample size unfortunately precluded adjustment for more than two covariates. Note that these were selected a priori based on scientific grounds (i.e., longer duration of PTSD might more severely impact liver function and antidepressants would increase liver enzymes). With the sample size at hand, we were already slightly below the minimum ratio of 10:1 for participants to observed variables in models that included three dependent variables. This ratio is commonly recommended to prevent overfitted models with biased partial correlation coefficients [36]. Particularly age and history of diabetes correlated with ALT in the bivariate analysis and might be important confounders, although analyses in larger samples will have to test this. In terms of variables that did not correlate with liver enzymes individually, these might explain the considerable variance of relationships if aggregated and applied to a larger sample. We are unable to state which amount of enzyme activity is of hepatic origin and which quantity derives from other tissues. For instance, we did not assess isoforms of ALP, particularly its bone fraction. Moreover, AST is less specific for hepatic damage than ALT. This might partially explain why the total PTSD symptom score assessed at study entry predicted ALT but not AST. We investigated liver enzymes in relation to PTSD that had occurred post-MI. Whether similar relationships are found in healthy individuals and medical patients with PTSD that emerged after different types of trauma remains to be seen. The clinical relevance of our findings might be challenged by the fact that only a few patients exceeded the upper reference range for enzyme levels; however, virtually all patients had statins which, for instance, lower mildly elevated AST levels [49]. We did not perform liver biopsy and ultrasound to exclude chronic liver disease or biliary obstruction. Particularly, we did not conduct a background work-up for the presence of NAFLD, chronic viral hepatitis, and hemochromatosis. However, this drawback might be assumed to not have inflated substantial bias into our findings, since there is currently no evidence that these rather rare conditions should be overly prevalent in patients with PTSD. We also did not measure enzyme levels at study entry and therefore we were unable to estimate the relationship between trajectories in PTSD and liver enzyme levels over time. This weakness of the study design evidently limits our estimate of a prospective association between PTSD symptomatology and liver enzymes.

To sum up, we found that PTSD severity following MI was associated with elevation of plasma levels of liver enzymes, suggesting that chronic psychological stress predicts liver injury in humans. Given that mildly elevated levels of liver enzymes are markers of hepatic and systemic inflammation alike, our findings provide one psychobiological mechanism by which PTSD might contribute to CVD. To further elucidate such a theory, future studies may want to investigate whether NFALD shows an association with chronic psychological stress and PTSD in particular. To better understand the biology of PTSD, it also seems worthwhile to investigate whether liver enzymes mediate or moderate an association between NFALD and CVD in healthy individuals and post-MI patients with PTSD.

Acknowledgments The authors are grateful to Sabrina Imhasly, Cédric Lehner, Annette Kocher, Verena Mast, Edith Matz, and Iris Stamm for technical and editorial support.

\section{References}

1. Pratt DS, Kaplan MM. Evaluation of abnormal liver-enzyme results in asymptomatic patients. $N$ Engl J Med. 2000;342: 1266-1271.

2. Schmidt FW, Schmidt E. Progress in the enzyme diagnosis of liver disease: reality or illusion? Clin Biochem. 1990;23:375-382.

3. Harris H. The human alkaline phosphatases: what we know and what we don't know. Clin Chim Acta. 1990;186:133-150. 
4. Sanchez O, Viladrich M, Ramírez I, Soley M. Liver injury after an aggressive encounter in male mice. Am J Physiol Regul Integr Comp Physiol. 2007;293:R1908-R1916.

5. Salas M, Tuchweber B, Kourounakis P. Liver ultrastructure during acute stress. Pathol Res Pract. 1980;167:217-233.

6. Matsuoka N, Arakawa H, Kodama H, Yamaguchi I. Characterization of stress-induced sudden death in cardiomyopathic hamsters. J Pharmacol Exp Ther. 1998;284:125-135.

7. Adachi S, Kawamura K, Takemoto K. Oxidative damage of nuclear DNA in liver of rats exposed to psychological stress. Cancer Res. 1993;53:4153-4155.

8. Chida Y, Sudo N, Kubo C. Does stress exacerbate liver diseases? J Gastroenterol Hepatol. 2006;21:202-208.

9. Fukudo S, Suzuki J, Tanaka Y, Iwahashi S, Nomura T. Impact of stress on alcoholic liver injury; a histopathological study. J Psychosom Res. 1989;33:515-521.

10. Irie M, Tsutsumi A, Shioji I, Kobayashi F. Effort-reward imbalance and physical health among Japanese workers in a recently downsized corporation. Int Arch Occup Environ Health. 2004;77:409-417.

11. Yehuda R. Post-traumatic stress disorder. N Engl J Med. 2002; 346:108-114.

12. Tedstone JE, Tarrier N. Posttraumatic stress disorder following medical illness and treatment. Clin Psychol Rev. 2003;23: 409-448.

13. Schelling G. Post-traumatic stress disorder in somatic disease: lessons from critically ill patients. Prog Brain Res. 2008;167: 229-237.

14. Gurevich M, Devins GM, Rodin GM. Stress response syndromes and cancer: conceptual and assessment issues. Psychosomatics. 2002;43:259-281.

15. Spindler H, Pedersen SS. Posttraumatic stress disorder in the wake of heart disease: prevalence, risk factors, and future research directions. Psychosom Med. 2005;67:715-723.

16. Guler E, Schmid JP, Wiedemar L, Saner H, Schnyder U, von Känel R. Clinical diagnosis of posttraumatic stress disorder after myocardial infarction. Clin Cardiol. 2009;32:125-129.

17. Gander ML, von Känel R. Myocardial infarction and posttraumatic stress disorder: frequency, outcome, and atherosclerotic mechanisms. Eur J Cardiovasc Prev Rehabil. 2006;13:165-172.

18. Abbas CC, Schmid JP, Guler E, Wiedemar L, Begré S, Saner H, Schnyder U, von Känel R. Trajectory of posttraumatic stress disorder caused by myocardial infarction: a two-year follow-up study. Int J Psychiatry Med. 2009;39:359-376.

19. Boscarino JA. Posttraumatic stress disorder and physical illness: results from clinical and epidemiologic studies. Ann N Y Acad Sci. 2004;1032:141-153.

20. Boscarino JA. A prospective study of PTSD and early-age heart disease mortality among Vietnam veterans: implications for surveillance and prevention. Psychosom Med. 2008;70:668-676.

21. Shemesh E, Yehuda R, Milo O, et al. Posttraumatic stress, nonadherence, and adverse outcome in survivors of a myocardial infarction. Psychosom Med. 2004;66:521-526.

22. Kimerling R, Gima K, Smith MW, Street A, Frayne S. The Veterans Health Administration and military sexual trauma. Am J Public Health. 2007;97:2160-2166.

23. Brea A, Mosquera D, Martín E, et al. Nonalcoholic fatty liver disease is associated with carotid atherosclerosis: a case-control study. Arterioscler Thromb Vasc Biol. 2005;25:1045-1050.

24. Falck-Ytter Y, Younossi ZM, Marchesini G, McCullough AJ. Clinical features and natural history of nonalcoholic steatosis syndromes. Semin Liver Dis. 2001;21:17-26.

25. Wilson PW. Evidence of systemic inflammation and estimation of coronary artery disease risk: a population perspective. Am J Med. 2008;121(10 Suppl 1):S15-S20.
26. Oliveira AC, Oliveira AM, Almeida MS, et al. Alanine aminotransferase and high sensitivity C-reactive protein: correlates of cardiovascular risk factors in youth. J Pediatr. 2008;152: 337-342.

27. Kerner A, Avizohar O, Sella R, et al. Association between elevated liver enzymes and $\mathrm{C}$ reactive protein: possible hepatic contribution to systemic inflammation in the metabolic syndrome. Arterioslcer Thromb Vasc Biol. 2005;25:193-197.

28. von Känel R, Hepp U, Kraemer B, et al. Evidence for low-grade systemic proinflammatory activity in patients with posttraumatic stress disorder. J Psychiatr Res. 2007;41:744-752.

29. Schindhelm RK, Dekker JM, Nijpels G, et al. Alanine aminotransferase predicts coronary heart disease events: a 10-year follow-up of the Hoorn study. Atherosclerosis. 2007;191: 391-396.

30. Adibi P, Sadeghi M, Mahsa M, Rozati G, Mohseni M. Prediction of coronary atherosclerotic disease with liver transaminase level. Liver Int. 2007;27:895-900.

31. Stieglitz RD, Frommberger U, Foa EB, Berger M. Evaluation of the German version of the PTSD Symptom Scale (PSS). Psychopathology. 2001;34:128-133.

32. Foa EB, Cashman L, Jaycox L, Perry K. The validation of a selfreport measure of posttraumatic stress disorder: the Posttraumatic Diagnostic Scale (PDS). Psychol Assess. 1997;9:445-451.

33. Blake DD, Weathers FW, Nagy LM, et al. The development of a clinician-administered PTSD scale. J Trauma Stress. 1995;8: 75-90.

34. Schnyder U, Moergeli $\mathrm{H}$. German version of clinician-administered PTSD scale. J Trauma Stress. 2002;15:487-492.

35. Schnyder U, Moergeli H, Trentz O, Klaghofer R, Buddeberg C. Prediction of psychiatric morbidity in severely injured accident victims at one-year follow-up. Am J Respir Crit Care Med. 2001; 164:653-656.

36. Babyak MA. What you see may not be what you get: a brief, nontechnical introduction to overfitting in regression-type models. Psychosom Med. 2004;66:411-421.

37. Perneger TV. What's wrong with Bonferroni adjustments? BMJ. 1998;316:1236-1238.

38. O’Donnell T, Hegadoren KM, Coupland NC. Noradrenergic mechanisms in the pathophysiology of post-traumatic stress disorder. Neuropsychobiology. 2004;50:273-283.

39. Yehuda R, Siever LJ, Teicher MH, et al. Plasma norepinephrine and 3-methoxy-4 hydroxyphenylglycol concentrations and severity of depression in combat posttraumatic stress disorder and major depressive disorder. Biol Psychiatry. 1998;44:56-63.

40. Blanchard EB, Kolb LC, Prins A, Gates S, McCoy GC. Changes in plasma norepinephrine to combat-related stimuli among Vietnam veterans with posttraumatic stress disorder. J Nerv Ment Dis. 1991;179:371-373.

41. Iwai M, Saheki S, Ohta Y, Shimazu T. Footshock stress accelerates carbon tetrachloride induced liver injury in rats: implication of the sympathetic nervous system. Biomed Res. 1986;7: $145-154$.

42. Kitamura H, Konno A, Morimatsu M, Jung BD, Kimura K, Saito M. Immobilization stress increases hepatic IL-6 expression in mice. Biochem Biophys Res Commun. 1997;238:707-711.

43. Jung BD, Kimura K, Kitamura H, et al. Norepinephrine stimulates interleukin- 6 mRNA expression in primary cultured rat hepatocytes. J Biochem. 2000;127:205-209.

44. Meewisse ML, Reitsma JB, de Vries GJ, Gersons BP, Olff M. Cortisol and post- traumatic stress disorder in adults: systematic review and meta-analysis. Br J Psychiatry. 2007;191:387-392.

45. Swain MG. Stress and the gastrointestinal tract. I. Stress and hepatic inflammation. Am. J Physiol Gastrointest Liver Physiol. 2000;279:G1135-G1138. 
46. Athyros VG, Kakafika AI, Karagiannis A, Mikhailidis DP. Do we need to consider inflammatory markers when we treat atherosclerotic disease? Atherosclerosis. 2008;200:1-12.

47. Targher G, Marra F, Marchesini G. Increased risk of cardiovascular disease in non alcoholic fatty liver disease: causal effect or epiphenomenon? Diabetologia. 2008;51:1947-1953.

48. Canbakan B, Senturk H, Tahan V, et al. Clinical, biochemical and histological correlations in a group of non-drinker subjects with non-alcoholic fatty liver disease. Acta Gastroenterol Belg. 2007; 70:277-284.

49. Wiesinger HA, Shah J, White A, et al. Liver biochemistry abnormalities in a quaternary care lipid clinic database. Ann Hepatol. 2008;7:63-66. 